\title{
14 to 18-year-old Hungarian high-school students' view of mathematicians appearing in the media - a case study
}

\author{
Orsolya Dóra Lócska ANd Zoltán KovÁCS
}

Abstract. One way to develop positive attitude toward STEM subjects that popular media, including movies and films can be engaged to promote more positive and inclusive STEM images. The movie Hidden numbers offers an opportunity to explore the representations of scholars, especially mathematicians within a biographical drama. Focusing on 5 characters, this article first discusses whether these characters fit into stereotypical scientist image or not. Secondly, we examine how high school students evaluate these characters. We argue that this movie is suitable to promote positive attitude toward STEM subjects.

Key words and phrases: attitude toward STEM subjects, impact of movie in STEM education, students' view of mathematicians.

ZDM Subject Classification: C94.

\section{Introduction}

In the past several years, the major goals for STEM education have been stated many times and in various way, see eg. [1]. The development of science literacy, a positive attitude toward science, and the development of an ability to use the scientific methods are perhaps three of the major goals of science instruction for a long time. In this paper we focus on attitudes that students have toward science and more specially, toward mathematics. 
One way to develop positive attitude toward STEM subjects that popular media and toy retail outlets, including movies and films, television, and social media can be engaged to promote more inclusive STEM images [1].

The impact of the media on younger generations' value system is undeniable. This is one of the reasons why media education is getting more and more emphasized with the development and spread of technology. In this paper, we study a specific medium-mediated content.

The film, as one of the most popular media, features many new and exciting characters. Television and the movies also are increasingly focused on STEM content, characters, and storylines. Shows like Criminal Minds and movies like The Martian have demonstrated the entertainment value of science [4]. While on the surface, many of the science-based shows and movies undermine long-held stereotypes, they also often end up reinforcing the status quo about women and people of color in STEM in their ultimate goal to entertain and engage the public audience [11].

A historical, biographical drama 'Hidden Figures' presented in Hungary on 19 January 2017 displays several scholars, some of them can be identified as mathematicians. When seeing the movie for the first time, we found that 'Hidden Figures' is a work that is worth showing to students.

This article is guided by the following questions:

(1) Do the characters in the movie 'Hidden Figures' fit into the stereotypical image of the scholar?

(2) How do students relate to the characters appearing in the movie 'Hidden Figures', especially, how do they see the temperament of characters?

\section{Theoretical underpinnings}

Among numerous studies devoted to the image of scholars, here we highlight the study [2] in which the author creates the 'draw-a-scientist' method. Based on this method the author outlines the stereotypical scientist: who is elderly or middle-aged, wears glasses and cloaks, is bald or has shaggy hair and some facial hair, and speaks incomprehensibly.

The same method was applied by Schilbeci and Sorensen [14] and later in Finson's [5] studies on different ethnic groups with different cultures. It was found that the opinions of students from different cultures are barely different. 
This uniform scientist stereotype was explained by the influence of the media. As for the students interviewed, the same media influence was detected.

Paper [12] treats the mathematician's image. Analogously to [2], the 'drawa-mathematician' method was applied for assessing the students' opinions. It was found that stereotypical mathematicians and scholars are hard to distinguish.

In his doctoral dissertation [7] Korándi studies the views of Hungarian mathematics major students about mathematicians' appearance. He found that students' views about mathematicians do not show any significant difference from other studies. Interestingly, however, the smiles in the drawings were more pronounced.

We have previously emphasized that the image of mathematicians is influenced by the media. The image of mathematicians emerging from movies is the subject of several studies. In [8] Korándi and Pluhár analyzed the characters of the movie 'Good Will Hunting' in comparison to the stereotyped scholar image. It was found that the characters appearing in the film partially reinforce the existing stereotypes, but contradict in some respects, for example, they are mostly younger. They concluded that 'In the movie the joy of doing mathematics is not present at all. [...] It seems from the movie that doing mathematics is a very tiring and boring activity. Therefore, the mathematician characters are not attractive figures, and nobody would want to be like them.'

The series 'NUMB3RS', started in 2005 is a milestone in forming the mathematician's image [7]. It gives the impression that a mathematician is young, handsome, humorous, intelligent and has social relationships. This tendency seems to be continued. In the previous work of the first author she reports a study in which the images of the mathematician and mathematics were observed in nine Hungarian media from September to November in 2016 [9]. By qualitative content analysis [15] 144 records were categorized; 100 of them refer to mathematics and 44 refer to mathematicians. Codes referring to mathematicians were developed in an inductive way, see Table 1. Three of the four codes (35 are for appearances out of 44) undoubtedly reflect positive judgements (intelligent, humane, an attractive carrier option). 


\begin{tabular}{lllll}
\hline $\begin{array}{l}\text { A } \\
\text { mathemat- } \\
\text { ician is . . }\end{array}$ & intelligent & $\begin{array}{l}\text { extrava- } \\
\text { gant }\end{array}$ & humane & $\begin{array}{l}\text { an attractive ca- } \\
\text { reer option }\end{array}$ \\
\hline $\begin{array}{l}\text { Number of } 10 \\
\text { records }\end{array}$ & 9 & 17 & 8 \\
\hline
\end{tabular}

Table 1. The mathematicians' image in nine Hungarian media in 2016

The image of mathematics was also positive in the media studied. The nature of mathematics was described by the following codes 'cultural heritage', 'special mental process', 'creative activity', 'the source of joy ', 'the source of order and aesthetic in the patterns', 'independent science', 'helps other professions', 'usable in everyday life'. Items 'the source of joy' and 'usable in everyday life' made almost half of the codes (47 out of 100).

Another important question is the appearance of genders in movies. The stereotyped mathematician is male, but this tendency is changing nowadays. While in 'Good Will Hunting' only one female scientist appears, in 'The Big Bang Theory' (also analysed in [9]) four male and two female scientists are presented. Moreover, three female and two male mathematicians appear in 'Hidden Figures' as main characters, which reverses the proportions.

\section{Research Method}

So as to answer the first research question, we apply the character analysis method used by Korándi and Pluhár [8]. The authors use eight categories to describe characters in 'Good Will Hunting': age, gender, race, build, facial hair, glasses, clothing, hair. Concerning the categories they used the codes summarized in Table 2 .

The method for investigating the second research question was the interview. The purpose of the interview is to reveal the students' opinions and first impressions on math characters in 'Hidden Figures'. 


\begin{tabular}{ll}
\hline category & code \\
\hline age & young, middle-aged, elderly \\
gender & male, female \\
race & white, black, Hindu \\
build & slim, overweight \\
facial hair & no, moustache, beard \\
glasses & no, yes, reading glasses \\
clothing & casual, formal, old-fashioned \\
hair & usual, balding, unsuitable \\
\hline
\end{tabular}

Table 2. Categories and codes used in [8] for character analysis

Although the film contains a lot of humorous scenes, it tells a serious story. Therefore, the age group of primary-school students would probably be less interested in the movie. The story is more likely to attract the high-school age group. So we decided on the age group of 14 to 18 (grades 9 to 12 in Hungary) to be interviewed. The questionnaire was filled in by a total of 117 high-school students (see Table 3.) The students came from the same urban school.

\begin{tabular}{lllll}
\hline grade & 9 & 10 & 12 \\
\hline $\begin{array}{l}\text { number } \\
\text { students }\end{array}$ & of & 47 & 44 & 26 \\
\hline
\end{tabular}

Table 3. Number of students in the survey

Luchins argues that the first encounter, or first impression, definitely influences the judgements [10]. This phenomenon is called the 'priority effect' [6]. Therefore, we decided to cut out scenes with the first appearances of the characters. Thus, a nearly 20-minute extract was created, which is short enough to keep the attention, but long enough for the viewers to get to know the characters (see the Appendix of this paper).

Primarily, we wanted to define the characteristics and temperaments of the characters through the eyes of the students. We also found the likeability of the characters an interesting issue, especially in connection with their temperaments.

For character description we started with three of four categories used by [9], i.e. 'intelligent', 'extravagant', 'humane', see also Table 2. The category 'absentminded' is one of the decisive features of the stereotyped scholar, and the category 'happy' was added to the questionnaire following the comment by Korándi and 
Pluhár: 'The mathematicians seem to be happy only on a few pictures, ...' [8]. The eighth category 'likeable' refers to the sympathy for the character.

Students answered the question 'To what extent does the given category characterise the person?' on Likert scale $(1=$ not in the least, $5=$ strongly characteristic.)

Cloninger in [3] argues that the temperament is an individual pattern of automatic responses that shape habits and moods. Seven temperament types were identified in [13]: independent, reliable, systematic, careful, daring, passionate, impulsive, sensitive.

Students were free to select the typical temperament of the given character from the above eight types.

The interview was conducted with paper-based questionnaires.

\section{Results}

\section{Character Analysis}

We described five main characters from 'Hidden Figures' by the dimensions used by Korándi and Pluhár in [8]. The selected characters: Katherine G. Johnson, Dorothy Vaughan, Mary Jackson, Al Harrison, Paul Stafford, see Table 4.

\begin{tabular}{llllll}
\hline & Katharine & Dorothy & Mary & Al & Paul \\
\hline age & young & young & young & middle-aged & young \\
gender & female & female & female & male & male \\
race & black & black & black & white & white \\
build & normal & plump & slim & normal & normal \\
facial hair & - & - & - & no & no \\
glasses & yes & no & no & yes & no \\
clothing & neat & neat & neat & formal & casual \\
hair & tended & tended & tended & tended & tended \\
\hline
\end{tabular}

Table 4. Character analysis of the mathematicians in 'Hidden Figures'

The existence of a female mathematician stands out in itself from the stereotypical image [7]. The three ladies are well dressed and nicely feminine, and their hairstyle is groomed. Only two actors wear a pair of glasses but no cloak. 
As we have earlier argued, while talking about the new image of the mathematician, social aspects came up. Watching the movie, we can get an insight into the characters' family lives. Women's family relationships are well displayed, but the viewer gets less information about the family background of male characters. Female mathematicians are all mothers, but as for the man only Al's wife is mentioned.

Female scientists are not only starring, but contradict almost every feature of the stereotypical image, of course, with the exception of high intelligence. As for $\mathrm{Al}$ and Paul, there is a shift away from stereotypes, but not to the same extent as for ladies.

\section{Interviews}

We report the results of the Likert scale questions based on the most frequent answers, see Figure 1.

Students found that each character's intelligence is very high. Probably most students thought that 'Everyone works for the NASA, so they're all smart', as one of the students reported.

Students do not think that extravagance is a typical trait of the main characters. More precisely, concerning two characters the most frequent answer is 3, which means that the students are uncertain about this.

With regard to absent-mindedness, similar results can be observed: characters are not regarded as absent-minded researchers.

Students considered ladies as more humane then gentlemen. Responses might have been affected by the family background described above. In the case of $\mathrm{Al}$ and Paul, almost no information is given to the viewer about their human relationships. We found similar results about likeability, which may correlate with the question of being happy.

It is possible to motivate students with likeable characters, and the mathematician's profession can represent an attractive option, if students see happy characters. Both features - likeability and happiness - are influenced by the temperament characterizing the person. The most frequent typical temperaments were 'reliable' and 'systematic' in the students answers (Table 5.)

During the character analysis, it became clear that female characters stand in opposition to the stereotyped scholar images, while male characters are slightly different from them. We got the same results from the students' opinions. Thus, the impact of the movie on the students' views was coherent with the results of our character analysis. 


\begin{tabular}{llll}
\hline character & typical temperament & happiness & likeability \\
\hline Katherine & reliable & 4 & 4 \\
Dorothy & reliable & 4 & 4 \\
Mary & daring & 4,5 & 4 \\
Al & systematic & 3 & 3 \\
Paul & systematic, careful & 3 & 3 \\
\hline
\end{tabular}

Table 5. Temperament, happiness and likeability

\section{Discussion}

Our research is a case study. Our findings cannot be considered as representative surveys of the media or student age groups, it can only be applied to the examined medium and the examined group. In addition, a nearly 20-minute extract of 'Hidden Figures' is not equivalent to the entire film.

\section{Findings}

Based on the students' answers, trustworthy and reckless characters seemed happy and sympathetic. A great deal of media can be useful to introduce scholars and mathematicians during the lessons that are highly characterized by one of these two temperaments. Because sympathetic characters are much more motivated by students, or if the mathematician is happy to do his job, he also looks at the mathematical field as positive. They found those characters that were more sympathetic than the stereotype scientist. One of the reasons for this may be that they received much more information about these actors, they were seen in more life situations.

\section{Summary}

We studied 14-18-year-old students' opinions on five scientists appearing in 'Hidden Figures'. Our results re-enforce the statements that a new, attractive image of the mathematician is appearing in the media.

Our results, according to the research questions. 
(1) Do the characters in the movie 'Hidden Figures' fit into the stereotypical image of the scholar? Three of the main characters stand clearly in opposition to the stereotyped scholar image. They are young mothers who are pretty dressed and care for their appearance. Of the former stereotypes, only the glasses are displayed, with only one character. As for the male characters, there is a shift away from stereotypes, but to a lesser extent than for the ladies.

(2) How do students relate to characters that appear in the movie 'Hidden Figures', especially, how do they see the temperament of the characters?

The 'shift away from stereotypes' effect is definitely detectable in the students' views about the main characters. According to the students, the characters are clearly intelligent, while they are not extravagant or absent minded. They consider the female characters as happy, and they do not think that the male characters are unhappy. The positive opinion on happiness results in the likeability of the character according to our research.

\section{Appendix: Selected Scenes}

First appearance I. (00:03:08-00:12:00, 8 minutes 52 second) In these scenes you can first see all the main characters involved in the study. During this interval all the characters speak, and the characters' positions at NASA are revealed.

First appearance II. (00:16:37-00:20:17, 3 minutes 40 seconds) Three of the examined characters are included in this section; the two male characters interact with Katherine. They talk about the purpose and the difficulties of their work at NASA.

First Family Scene I. (00:32:20-00:33:28, 1 minute 8 seconds) This is the scene where all the three female characters appear together with their families in the congregation.

Scene of discovery (01:31:46-01:33:27, 1 minute 41 seconds) We can see Katherine's solution to a mathematical problem in a large office. Compared to the first appearance, there is sharp contrast between the characters' communication and interpersonal relations. One can see Katherine's growing professional recognition.

First Family Scene II. (01:39:37-01:40:40, 1 minute 3 seconds) We get almost no information about the family lives of the two men. In this scene a person refers to Al's wife. 
Last scenes (01:57:14-01:59:10, 1 minute 56 seconds) The film is based on a true story, so at the end there is review on the lives of the main characters. The viewer can get accurate information about their professional advancement.

\section{Acknowledgments}

The first author was supported by the ÚNKP-17-2 New National Excellence Program of the Ministry of Human Capacities.

\section{References}

[1] STEM 2026, A Vision for Innovation in STEM Education. Department of Education, USA, 2016.

[2] D. W. Chambers, Stereotypic images of the scientist: The draw-a-scientist test, Science Education (1983), 255-265.

[3] C. S. Cloninger, D. M. Svrakic, and T. R. Przybeck, A psychobiological model of temperament and character, Arch. Gen. Psychiatry (1993), 975-990.

[4] J. Coody Smits, How science informs the stories on Hollywood's silverscreen. Retrieved from http://www.educationandcareernews.com/stem/how-scienceinforms-the-stories-onhollywoods-silver-screen, 2016.

[5] K. D. Finson, J. B. Beaver, and B. L. Cramond, Development and eld test of a checklist for the draw-a-scientist test, Scool Science and Mathematics (1995), 195-205.

[6] J. Forgas, Interpersonal behaviour: the psychology of social interaction, Pergamon Press, 1985.

[7] J. Korándi, Investigating Mathematics and Media Relations, PhD thesis, University of Debrecen, 2012, in Hungarian.

[8] J. Korándi and G. Pluhár, Mathematics in Good Will Hunting I: The mathematicians in Good Will Hunting, Teaching Mathematics and Computer Science (2012), $375-388$.

[9] O. D. Lócska, Mathematics in the media, media in mathematics education Retrieved from https://www.slideshare.net/secret/BYkMdzRQjUPM8B, 2017, XXXIII. OTDK, in Hungarian.

[10] A. S. Luchins, Experimental attempts to minimize the impact of first impression, Yale University Press, 1957.

[11] H. McIntosh, Representations of female scientists in The Big Bang Theory, Journal of Popular Film and Television 42 (2014), 195-204. 
[12] S. H. Pickers and J. S. Berry, Investigating pupils' images of mathematicians, Education Studies in Mathematics (2000), 65-94.

[13] S. Rózsa, J. Kállai, A. Osváth, and M. Bánki, Temperaments and Character. Cloninger's Psycho-biological Model, Medicina Kiadó, Budapest, 2005, in Hungarian.

[14] R. Schilbeci and I. Sorensen, Elementary school children's perceptions of scientists, School Science and Mathematic (1983), 14-20.

[15] M. Schreier, Qualitative Content Analysis in Practice, Sage, 2012.

ZOLTÁN KOVÁCS

DEPARTMENT OF MATHEMATICS AND COMPUTER SCIENCE

UNIVERSITY OF NYÍREGYHÁZA

NYÍREGYHÁZA, HUNGARY

E-mail: kovacs.zoltan@nye.hu

ORSOLYA DÓRA LÓCSKA

KÖLCSEY FERENC REFORMÁTUS GYAKORLÓ ÁLTALÁNOS ISKOLA

DEBRECEN 


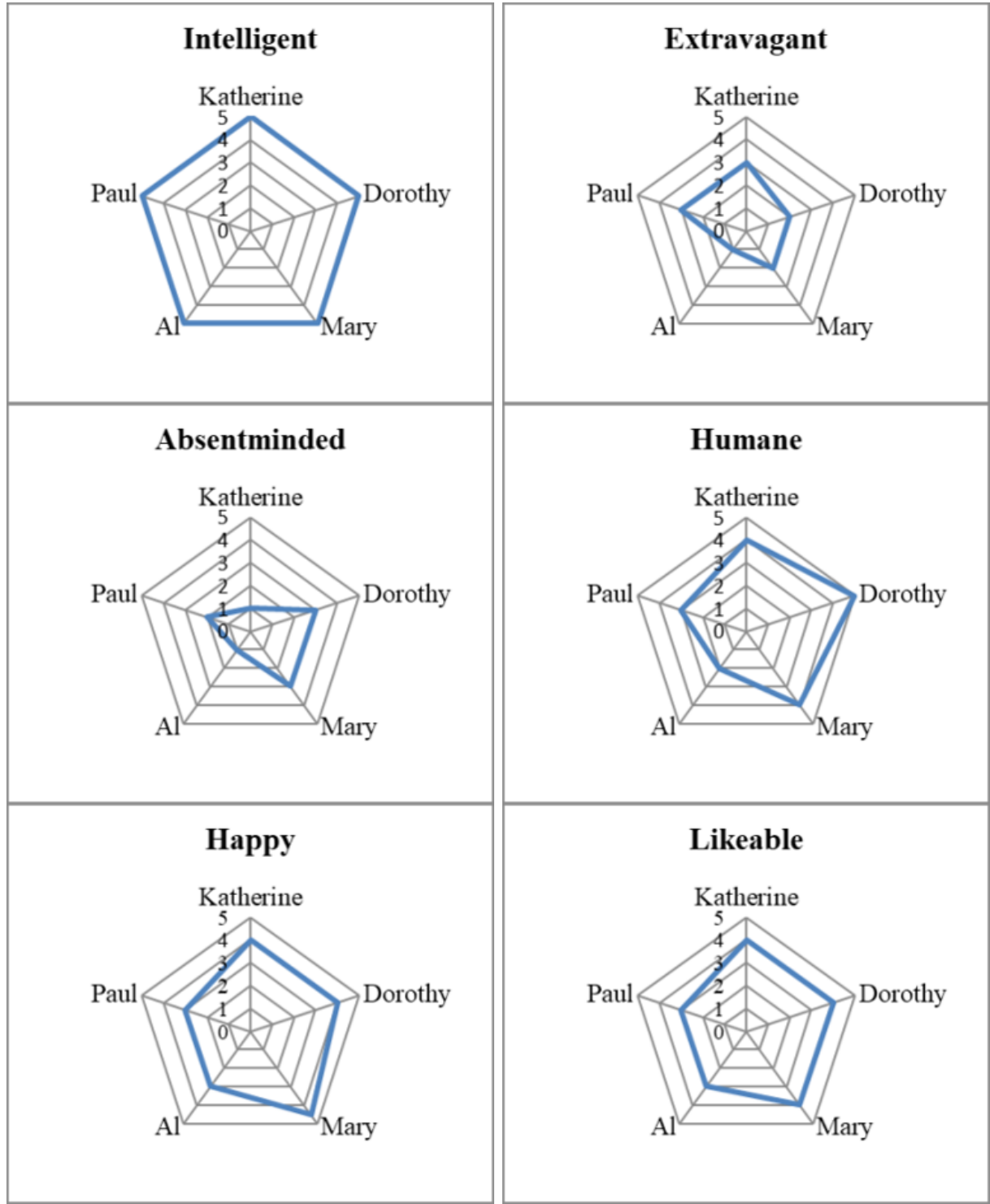

Figure 1. Students' most frequent answers describing the nature of characters (Received January, 2018) 